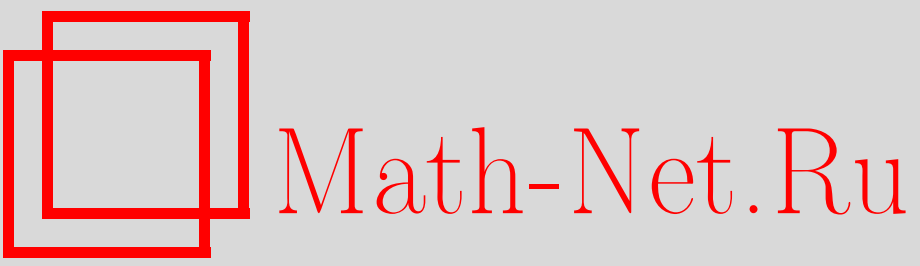

Д. П. Санкович, О некоторых свойствах функциональных интегралов по мере Боголюбова, ТМФ, 2001, том 126, номер 1, 149-163

DOI: https://doi.org/10.4213/tmf421

Использование Общероссийского математического портала Math-Net.Ru подразумевает, что вы прочитали и согласны с пользовательским соглашением

http://www.mathnet.ru/rus/agreement

Параметры загрузки:

IP : 3.85 .183 .62

26 апреля 2023 г., 17:20:20 
ТЕОРЕТИЧЕСКАЯ

И МАТЕМАТИЧЕСКАЯ

ФИЗИКА

Том 126, № 1

январь, 2001

(C) 2001 г

Д. П. Санкович*

\title{
О НЕКОТОРЫХ СВОЙСТВАХ ФУНКЦИОНАЛЬНЫХ ИНТЕГРАЛОВ ПО МЕРЕ БОГОЛЮБОВА
}

\begin{abstract}
Рассмотрены вопросы интегрирования по мере Боголюбова в пространстве непрерывных функций. Вычислены некоторые функциональные интегралы по этой мере. Построены приближенные формулы, точные для функциональных многочленов заданной степени, а также формулы, точные для интегрируемых функционалов более широкого класса. Доказано неравенство для следов и получена оценка сверху для гиббсовского равновесного среднего от квадрата оператора координаты в случае одномерного нелинейного осциллятора с положительным симметричным взаимодействием.
\end{abstract}

\section{1. ВВЕДЕНИЕ}

Вопросами интегрирования функций, заданных на абстрактных множествах, начал заниматься Фреше [1], обобшив соответствуюшим образом метод Лебега. Несколько позднее эти проблемы изучались Даниелем [2], [3], которьй воспользовался идеей продолжения линейных функционалов. Основой его теории является совокупность $H(X)$ элементарных функций $h(x)$ на множестве $X$ с заданным для них элементарным интегралом $I(h)$. При выполнении некоторых условий эта совокупность может быть расширена до некоторой совокупности $L$, на которую распространяется интеграл $I$, причем так, что $L$ представляет собой банахово пространство с нормой $\|\varphi\|=I(|\varphi|)$. В этом и состоит построение интеграла Лебега в схеме Даниеля [4], [5].

С теорией интеграла Даниеля много обшего имеют ранние результаты Винера [6], который определил процесс интегрирования функционалов и показал, что рассмотренный им интеграл является интегралом Даниеля. Следует отметить, что во всех работах Винера начиная с 1921 года задача функционального интегрирования связывается с изучением броуновского движения частиц. На сегменте $[0,1]$ определяется множество непрерывных действительных функций $C=C[0,1]$, удовлетворяюших условию $x(0)=0$. При этом $x(t)$ является координатой частицы, совершаюшей броуновское движение вдоль оси под действием случайных толчков и находящейся при $t=0$ в начале координат. Винеровская мера имеет нулевое среднее и корреляционную функцию $\min (t, s)$. Эта мера включается в более обший класс мер в функциональных пространствах, которые принято называть гауссовыми.

\footnotetext{
* Математический институт им. В. А. Стеклова РАН, Москва, Россия
} 
Впервые функциональное интегрирование в квантовой физике было применено Фейнманом [7]. Конструкция функционального (континуального) интеграла Фейнмана имеет некоторые общие свойства с интегралом Винера. Однако эти интегралы существенно различаются [8].

Идея записи физических наблюдаемых в виде континуальных интегралов получила свое развитие в квантовой теории поля для представления функций Грина. В свое время почти одновременно возникли два способа такого представления. Один из них основывался на формальном интегрировании уравнений в вариационных производных для функций Грина [9]-[12]. Боголюбовым был развит другой путь [13], который исходил из представления функций Грина через вакуумные ожидания хронологических произведений, причем операция усреднения по бозонному вакууму интерпретировалась как функциональный интеграл. В работе [14] боголюбовский способ функционального интегрирования был применен к вопросам градиентных преобразований электродинамических функций Грина и к исследованию модели Блоха-Нордсика. В дальнейшем Боголюбов вернулся к этому построению уже в рамках статистической механики при исследовании модели полярона [15]. В работе [16] было показано, что мера, возникающая в подходе Боголюбова, является гауссовой мерой в соответствуюшем пространстве непрерывных функций. Гиббсовские равновесные средние от хронологических произведений операторов выражаются в виде функциональных интегралов по данной мере.

Ниже рассмотрены некоторые свойства функциональных интегралов по мере Боголюбова. При этом используются методы теории гауссовых мер в банаховых пространствах [17], [18] и теории приближенного вычисления интегралов по гауссовым мерам [19], [20]. В разделе 2 приведены основные результаты теории интегрирования в абстрактных пространствах в приложении к конкретному случаю боголюбовской меры. В разделе 3 вычислены некоторые простейшие функциональные интегралы по мере Боголюбова. В разделе 4 рассмотрены формулы приближенного интегрирования. В разделе 5 доказано одно неравенство для следов, применяемое в теории фазовых переходов. Основное внимание в статье уделено приложению методов функционального интегрирования к задачам равновесной квантовой статистической механики.

\section{2. МЕРА БОГОЛЮБОВА В ПРОСТРАНСТВЕ НЕПРЕРЫВНЫХ ФУНКЦИЙ}

В работе [16] показано, что в пространстве $X=C^{\circ}[0, \beta]$ непрерывных на отрезке $[0, \beta]$ функций с равномерной метрикой $\rho=\max _{t \in[0, \beta]}|x(t)-y(t)|$, удовлетворяющих условию $x(0)=x(\beta)$, определена гауссова мера $\mu_{\text {Б }}$ с нулевым средним и корреляционной функцией

$$
B(t, s)=\frac{1}{2 m \omega \operatorname{sh} \frac{\beta \omega}{2}} \operatorname{ch}\left(\omega|t-s|-\frac{\beta \omega}{2}\right) .
$$

На пространстве с мерой $\left\{X, G, \mu_{\text {Б}}\right\}$, где $G$-выделенная на пространстве $X \sigma$-алгебра его подмножеств, рассматриваются измеримые функционалы $F(x)$. Тогда для гиббсовского равновесного среднего от $T$-произведения, взятого по гамильтониану $\widehat{\Gamma}$ гармони- 
ческого осциллятора, имеет место формула

$$
\langle T[F(\widehat{Q}(t))]\rangle_{\widehat{\Gamma}}=\int_{X} F(x(t)) d \mu_{\mathrm{Б}}(x),
$$

где интеграл понимается как интеграл Даниеля по пространству $X$,

$$
\widehat{\Gamma}=\frac{\hat{p}^{2}}{2 m}+\frac{m \omega^{2}}{2} \hat{q}^{2}, \quad \widehat{Q}(t)=e^{t \widehat{\Gamma}} \hat{q} e^{-t \widehat{\Gamma}}, \quad\langle\ldots\rangle_{\widehat{\Gamma}}=\frac{\operatorname{tr}\left(\ldots e^{-\beta \widehat{\Gamma}}\right)}{\operatorname{tr} e^{-\beta \widehat{\Gamma}}}
$$

величины $\hat{q}, \hat{p}$ представляют собой соответственно операторы координаты и импульса частицы с массой $m$ и удовлетворяют коммутационному соотношению $[\hat{q}, \hat{p}]=i$ (предполагается, что $\hbar=1), \beta$ - обратная температура, $\omega$ - собственная частота осциллятора $(\beta>0, \omega>0)$. Для интегрируемого функционала $F(x)$ среднее в формуле $(2)$ сушествует и конечно.

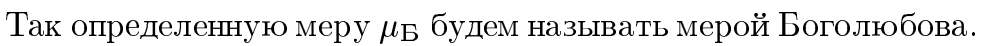

Ядро $B(t, s)$ корреляционного оператора $B$ симметрично и эрмитово. Оно принадлежит пространству $L^{2}$ функций двух переменных с суммируемым по мере Лебега квадратом в области $0 \leqslant t \leqslant \beta, 0 \leqslant s \leqslant \beta$. В силу теоремы Шмидта [5] всякая функция $A(t, s)$ с суммируемым квадратом, симметричная относительно своих аргументов, разлагается в смысле сходимости в среднем в ряд

$$
A(t, s)=\sum_{n} \lambda_{n} \Phi_{n}(t) \overline{\Phi_{n}(s)}
$$

где $\left\{\Phi_{n}(t)\right\}$ - ортонормированная последовательность собственных функций, а $\left\{\lambda_{n}\right\}-$ последовательность соответствуюших собственных значений оператора $A$, порожденного ядром $A(t, s)$. Для корреляционной функции (1) имеет место [16] разложение (3), где

$$
\Phi_{n}(t)=\frac{1}{\sqrt{\beta}} \exp \left(\frac{2 \pi i n t}{\beta}\right), \quad \lambda_{n}=\frac{1}{m} \frac{1}{\omega^{2}+\left(2 \pi n \beta^{-1}\right)^{2}}
$$

и $n$ пробегает все целые числа от $-\infty$ до $\infty$. По теореме Мерсера [5] ряд (3) для ядра $B(t, s)$ сходится равномерно, т.к. оператор $B$, порожденный ядром $B(t, s)$, положителен. Отметим также, что этот оператор вполне непрерывен. Разложение (3) для корреляционной функции $B(t, s)$ можно записать в пространстве действительных функций в виде

$$
B(t, s)=\sum_{n=-\infty}^{+\infty} \lambda_{n} \varphi_{n}(t) \varphi_{n}(s)
$$

где

$$
\varphi_{n}(t)= \begin{cases}\sqrt{\frac{2}{\beta}} \cos \frac{2 \pi n t}{\beta}, & n>0 \\ \sqrt{\frac{2}{\beta}} \sin \frac{2 \pi n t}{\beta}, & n<0 \\ \sqrt{\frac{1}{\beta}}, & n=0\end{cases}
$$


Сопряженное к $X$ пространство $X^{\prime}=V_{0}[0, \beta]$ есть пространство функций ограниченной вариации на $[0, \beta]$, удовлетворяющих условиям

$$
g(0)=0, \quad g(t)=\frac{1}{2}[g(t+0)+g(t-0)] \text { для } t \in(0, \beta) .
$$

В силу теоремы Рисса [5] линейные функционалы в $X$ имеют вид

$$
\langle\varphi, x\rangle=\int_{0}^{\beta} x(t) d \varphi(t),
$$

где интеграл понимается как интеграл Стильтьеса и $x(t) \in X, \varphi(t) \in V_{0}[0, \beta]$. Koppeляционный функционал в пространстве $X^{\prime}$ можно записать в виде

$$
K(\varphi, \psi)=\int_{0}^{\beta} \int_{0}^{\beta} B(t, s) d \varphi(t) d \varphi(s)
$$

где корреляционная функция меры имеет вид

$$
B(t, s)=\int_{X} x(t) x(s) d \mu(x) .
$$

В силу теоремы Кьюлбса [21] гильбертово пространство $H$,порожденное мерой $\mu$,представляет собой линейную оболочку собственных функций $\left\{\varphi_{n}(t)\right\}$ ядра $B(t, s)$, замкнутую относительно нормы, соответствуюшей скалярному произведению

$$
(x, y)_{H}=\sum_{n=-\infty}^{+\infty} \frac{1}{\lambda_{n}}\left(\int_{0}^{\beta} x(t) \varphi_{n}(t) d t\right)\left(\int_{0}^{\beta} y(t) \varphi_{n}(t) d t\right) .
$$

Функции $\left\{e_{n}(t)=\sqrt{\lambda_{n}} \varphi_{n}(t)\right\}_{n=-\infty}^{+\infty}$ образуют базис в пространстве $H$, и для почти всех $x \in X$ имеет место разложение

$$
x(t)=\sum_{n=-\infty}^{+\infty}\left(\int_{0}^{\beta} x(t) \varphi_{n}(t) d t\right) \varphi_{n}(t) .
$$

Общий вид линейного измеримого функционала на $X$ определяется выражением

$$
(a, x)=\sum_{n=-\infty}^{+\infty} \frac{1}{\lambda_{n}}\left(\int_{0}^{\beta} x(t) \varphi_{n}(t) d t\right)\left(\int_{0}^{\beta} a(t) \varphi_{n}(t) d t\right)
$$

где $a \in H, x \in X$. Полную ортонормированную систему в $H$ образуют также функции

$$
e_{n}(t)=\int_{0}^{\beta} B_{1 / 2}(t, u) \alpha_{n}(u) d u
$$

где $B_{1 / 2}(t, u)$ - ядро оператора $B^{1 / 2}, \alpha_{n}(t)$ - произвольная полная ортонормальная система в пространстве $L_{2}[0, \beta]$.

Отметим, что замыкание $\bar{H}$ гильбертова пространства $H$ является носителем меры $\mu$ и оно плотно почти всюду в $X[22]$. Тройка $(X, H, \mu)$ называется абстрактным винеровским пространством, а мера $\mu$ - абстрактной мерой Винера [23].

Заметим также, что в случае меры Боголюбова функция $G(t, s)=-m B(t, s)$ является функцией Грина краевой задачи

на отрезке $[0, \beta]$.

$$
\left\{\begin{array}{l}
y^{\prime \prime}-\omega^{2} y=0 \\
y(0)=y(\beta) \\
y^{\prime}(0)=y^{\prime}(\beta)
\end{array}\right.
$$




\section{3. ФУНКЦИОНАЛЬНЫЙ ИНТЕГРАЛ ПО МЕРЕ БОГОЛЮБОВА}

Пусть $a_{1}, a_{2}, \ldots, a_{n}$ - линейно независимые элементы из некоторого сепарабельного гильбертова пространства $H$, замыкание которого является носителем меры $\mu$ и которое плотно в $X$ почти всюду. Тогда

$$
\int_{X} F\left[\left(a_{1}, x\right),\left(a_{2}, x\right), \ldots,\left(a_{n}, x\right)\right] d \mu(x)=(2 \pi)^{-\frac{n}{2}} \frac{1}{\sqrt{\operatorname{det} A}} \int_{R^{n}} e^{-\frac{1}{2}\left(A^{-1} u, u\right)} F(u) d u
$$

если сушествует один из этих интегралов, где $A$-матрица с элементами $a_{i j}=\left(a_{i}, a_{j}\right)_{H}$; $i, j=1,2, \ldots, n ; u=\left(u_{1}, u_{2}, \ldots, u_{n}\right) ; d u=d u_{1} d u_{2} \ldots d u_{n}$. Если в качестве элементов $a_{j}$ взять ортонормированные векторы пространства $H$, то формула (4) принимает вид

$$
\int_{X} F\left[\left(a_{1}, x\right),\left(a_{2}, x\right), \ldots,\left(a_{n}, x\right)\right] d \mu(x)=(2 \pi)^{-\frac{n}{2}} \int_{R^{n}} e^{-\frac{1}{2}(u, u)} F(u) d u .
$$

Особенно простой вид будет у формулы (4) в случае, когда функционал $F(x)$ зависит только от значений функции $x(t)$ в конечном числе точек. Если, например,

$$
F(x(t))=x\left(t_{1}\right) x\left(t_{2}\right) \ldots x\left(t_{n}\right),
$$

то выполняется теорема Вика, в силу которой

$$
\int_{X} x\left(t_{1}\right) x\left(t_{2}\right) \ldots x\left(t_{n}\right) d \mu(x)=\sum B\left(t_{i_{1}}, t_{i_{2}}\right) B\left(t_{i_{3}}, t_{i_{4}}\right) \ldots B\left(t_{i_{2 k-1}}, t_{i_{2 k}}\right),
$$

где $n=2 k$ и суммирование проводится по всем $(2 k) ! /\left(2^{k} k !\right)$ способам разбиения чисел $1,2, \ldots, 2 k$ на $k$ различных неупорядоченных пар

$$
\left(i_{1}, i_{2}\right),\left(i_{3}, i_{4}\right), \ldots,\left(i_{2 k-1}, i_{2 k}\right) .
$$

При $n=2 k+1$ этот интеграл обращается в нуль. Так, в частности, в случае меры Боголюбова

$$
\begin{gathered}
\left\langle\hat{q}^{2}\right\rangle_{\widehat{\Gamma}}=\int_{X} x^{2}(t) d \mu_{\text {Б }}(x)=B(t, t)=\frac{1}{2 m \omega} \operatorname{cth} \frac{\beta \omega}{2} \\
\left\langle e^{a \hat{q}^{2}}\right\rangle_{\widehat{\Gamma}}=\sum_{n=0}^{\infty} \frac{(2 n) !}{2^{n}(n !)^{2}}\left(a\left\langle\hat{q}^{2}\right\rangle_{\widehat{\Gamma}}\right)^{n}=\frac{1}{\sqrt{1-\frac{a}{m \omega} \operatorname{cth} \frac{\beta \omega}{2}}},
\end{gathered}
$$

где в последней формуле необходимо считать, что $-m \omega \operatorname{th}(\beta \omega / 2) \leqslant a<m \omega \operatorname{th}(\beta \omega / 2)$.

Рассмотрим на $X$ квадратичный функционал

$$
A(x, x)=\sum_{k, j=1}^{\infty} a_{k j}\left(e_{k}, x\right)\left(e_{j}, x\right)
$$

где $a_{k j}=\left(A e_{k}, e_{j}\right)_{H}, A$ - самосопряженньй ядерный оператор, действующий из $H$ в $H$, $\left\{e_{k}\right\}_{k=1}^{\infty}-$ базис в $H$. Тогда, пользуясь формулой (4), можно вычислить следующие интегралы:

$$
\int_{X} A(x, x) d \mu(x)=\operatorname{tr} A, \quad \int_{X} A^{2}(x, x) d \mu(x)=(\operatorname{tr} A)^{2}+2 \sum_{k=1}^{\infty} \lambda_{k}^{2},
$$


где $\lambda_{k}$ - собственные значения оператора $A$. Имеет место также равенство

$$
\int_{X} e^{\frac{\lambda}{2} A(x, x)} d \mu(x)=\frac{1}{\sqrt{D_{A}(\lambda)}}
$$

где $D_{A}(\lambda)$ - характеристический определитель оператора $A$ в точке $\lambda$,

$$
\operatorname{Re} \lambda<\frac{1}{\lambda_{1}} \quad\left(\lambda_{1}>\lambda_{2}>\cdots\right),
$$

a

$$
\sqrt{D_{A}(\lambda)}=\sqrt{\left|D_{A}(\lambda)\right|} \exp \left[-\frac{i}{2} \arg D_{A}(\lambda)\right] .
$$

Выберем в формуле (5) в качестве квадратичного функционала

$$
A(x, x)=\int_{0}^{\beta} x^{2}(t) d t=\sum_{k=-\infty}^{\infty} \lambda_{k}\left(e_{k}, x\right)^{2},
$$

где $e_{k}=\sqrt{\lambda_{k}} \varphi_{k}$, а $\lambda_{k}$ и $\varphi_{k}$ - собственные значения и собственные функции ядра $B(t, s)$. Воспользуемся формулой [5]

$$
-\frac{d}{d \lambda} \ln D_{B}(\lambda)=\int_{0}^{\beta} B(t, t) d t+\lambda \int_{0}^{\beta} B^{(2)}(t, t) d t+\cdots+\lambda^{k} \int_{0}^{\beta} B^{(k)}(t, t) d t+\cdots,
$$

где $B^{(k)}$ - соответствуюшие итерированные ядра, которые в случае меры Боголюбова имеют вид

$$
B^{(k)}(t, t)=\frac{1}{\beta m^{k}} \sum_{n=-\infty}^{\infty} \frac{1}{\left[\omega^{2}+\left(2 \pi n \beta^{-1}\right)^{2}\right]^{k}} .
$$

Тогда

$$
\begin{aligned}
-\frac{d}{d \lambda} \ln D_{B}(\lambda) & =\frac{1}{\lambda} \sum_{n=-\infty}^{\infty} \sum_{k=1}^{\infty}\left(\frac{\lambda}{m}\right)^{k} \frac{1}{\left[\omega^{2}+\left(2 \pi n \beta^{-1}\right)^{2}\right]^{k}}= \\
& =\frac{\beta}{2 m} \frac{1}{\sqrt{\omega^{2}-\frac{\lambda}{m}}} \operatorname{cth}\left(\frac{\beta}{2} \sqrt{\omega^{2}-\frac{\lambda}{m}}\right),
\end{aligned}
$$

где $\lambda<m \omega^{2}$. Интегрируя последнее уравнение, получаем

$$
D_{B}(\lambda)=\frac{\operatorname{sh}^{2}\left(\frac{\beta}{2} \sqrt{\omega^{2}-\frac{\lambda}{m}}\right)}{\operatorname{sh}^{2} \frac{\beta \omega}{2}},
$$

откуда следует формула

$$
\int_{X} \exp \left(\frac{\lambda}{2} \int_{0}^{\beta} x^{2}(t) d t\right) d \mu_{\mathrm{Б}}(x)=\frac{\operatorname{sh} \frac{\beta \omega}{2}}{\operatorname{sh}\left(\frac{\beta}{2} \sqrt{\omega^{2}-\frac{\lambda}{m}}\right)}, \quad \lambda<m \omega^{2} .
$$


Заметим, что моменты

$$
m_{k}=\int_{X} A(x, x)^{k} d \mu_{\mathrm{Б}}(x)=\int_{X}\left(\int_{0}^{\beta} x^{2}(t) d t\right)^{k} d \mu_{\mathrm{Б}}(x)
$$

могут быть определены с помощью формулы (6) и равенства

$$
m_{k+1}=\left.2^{k+1} \frac{d^{k+1}}{d \lambda^{k+1}} \frac{1}{\sqrt{D_{B}(\lambda)}}\right|_{\lambda=0} .
$$

Если принять во внимание соотношение

$$
D_{B}(\lambda)=\prod_{n=-\infty}^{\infty}\left(1-\lambda \lambda_{n}\right)
$$

то из найденного выше значения определителя Фредгольма ядра $B(t, s)$ получается следуюшая формула для бесконечного произведения:

$$
\prod_{n=1}^{\infty}\left(1+\frac{a}{n^{2}+b^{2}}\right)=\frac{1}{\sqrt{1+\frac{a}{b^{2}}}} \frac{\operatorname{sh}\left(\pi b \sqrt{1+\frac{a}{b^{2}}}\right)}{\operatorname{sh}(\pi b)}, \quad a>-b^{2} .
$$

\section{4. ПРИБЛИЖЕННОЕ ВЫЧИСЛЕНИЕ ФУНКЦИОНАЛЬНЫХ ИНТЕГРАЛОВ}

Рассмотрим приближенные формулы, точные для функциональных многочленов заданной степени.

Пусть $X$ есть пространство $C[a, b]$ непрерывных на отрезке $[a, b]$ функций $x(t)$. Будем считать, что в $X$ задана некоторая гауссова мера $\mu$ с нулевым средним и корреляционной функцией $B(t, s)$. Произвольный непрерывный функциональный многочлен $P_{n}(x)$ степени $n$ на пространстве $C$ имеет вид

$$
P_{n}(x)=p_{0}+\sum_{j=1}^{n} \int_{a}^{b} \ldots \int_{a}^{b} x\left(t_{1}\right) \ldots x\left(t_{j}\right) d_{t_{1} \ldots t_{j}}^{(j)} g\left(t_{1}, \ldots, t_{j}\right),
$$

где $p_{0}=$ const, a остальные слагаемые представляют собой кратные интегралы Стильтьеса.

ТЕОРема 1 ([19]). Пусть $\nu$ - симметричная вероятностная мера на борелевских множествах из $R$, а заданная на $R \times[a, b]$ функиия $\rho(u, t)$ такова, что

1) $\rho(u, t)=-\rho(-u, t)$,

2) $\prod_{j=1}^{m} \rho\left(u, t_{j}\right) \in L(R, \nu)$ для $1 \leqslant m \leqslant 2 n+1$,

$$
\int_{-\infty}^{\infty} \rho(u, t) \rho(u, s) d \nu(u)=B(t, s)
$$

Тогда формула

$$
\int_{C} F(x) d \mu(x) \approx \int_{R^{n}} F\left(\theta_{n}(u, \cdot)\right) d \nu_{n}(u)
$$


әде функция

$$
\theta_{n}(u, t)=\sum_{j=1}^{n} c_{j} \rho\left(u_{j}, t\right)
$$

$c_{j}^{2}-$ корни многочлена

$$
Q_{n}(z)=\sum_{k=0}^{n} \frac{z^{n-k}}{k !}
$$

$\nu_{n}-$ мера в $R^{n}$, являющаяся п-кратныцм декартовым произведением мер $\nu$, точна для функциональных многочленов степени $2 n+1$.

Теорема 2 ([19]). Пусть выполнены условия теоремы 1. Тогда формула

$$
\begin{aligned}
\int_{C} F(x) d \mu(x) \approx & \frac{(-1)^{n}(A-n)^{n}}{n !} F(0)+ \\
& +\sum_{k=1}^{n}(-1)^{n-k} \frac{(A-n+k)^{n}}{k !(n-k) !} \int_{R^{k}} F\left(\theta_{k}^{(n)}(u, \cdot)\right) d \nu_{k}(u),
\end{aligned}
$$

əдe

$$
\begin{array}{cl}
\theta_{k}^{(n)}(u, t)=\frac{1}{\sqrt{A-n+k}} \sum_{j=1}^{k} \rho\left(u_{j}, t\right), & R^{k}=\underbrace{R \times R \times \cdots \times R}_{k}, \\
d \nu_{k}(u)=d \nu\left(u_{1}\right) \ldots d \nu\left(u_{k}\right), & k=1,2, \ldots, n,
\end{array}
$$

A - произвольная постоянная, точна для всех функииональных многочленов степени $2 n+1$.

В случае $A=n$ формула (9) принимает вид [24]

$$
\int_{C} F(x) d \mu(x) \approx I_{n}(F),
$$

где

$$
I_{n}(F)=\sum_{k=1}^{n}(-1)^{n-k} \frac{k^{n}}{k !(n-k) !} \int_{R^{k}} F\left(\theta_{k}(u, \cdot)\right) d \nu_{k}(u)
$$

и

$$
\theta_{k}(u, t)=\frac{1}{\sqrt{k !}} \sum_{j=1}^{k} \rho\left(u_{j}, t\right) .
$$

Нетрудно убедиться в справедливости следуюшего рекуррентного соотношения для величины $I_{n}(F)$ :

$$
I_{n}(F)=\frac{n^{n}}{n !} \int_{R^{n}} F\left(\theta_{n}(u, \cdot)\right) d \nu_{n}(u)-\sum_{k=1}^{n-1} \frac{k n^{n-1-k}}{(n-k) !} I_{k}(F) .
$$

Получение формул вида (8), (9) для приближенного вычисления интегралов по гауссовой мере связано с нахождением функшии $\rho(u, t)$ со свойствами 1-3 из теоремы 1 . При этом наиболее трудным является решение уравнения (7). 
Будем искать решение этого уравнения сначала для случая чисто дискретной меры $\nu$ на прямой. Напомним, что мера на прямой, сосредоточенная целиком на конечном или счетном множестве точек, называется дискретной.

Пусть на отрезке $[a, b]$ задано конечное или счетное число точек $\left\{x_{n}\right\}_{n=-\infty}^{\infty}$ и пусть каждой точке $x_{n}$ поставлено в соответствие положительное число $h_{n}$, причем

$$
\sum_{n} h_{n}<\infty
$$

Определим функцию $f$ на $[a, b]$, положив $f(x)=\sum_{x_{n}<x} h_{n}$. Функция $f(x)$ не убывает и непрерывна слева. Если точка $x$ совпадает с одной из точек $x_{n}$, скажем $x=x_{n_{0}}$, то

$$
f\left(x_{n_{0}}+0\right)-f\left(x_{n_{0}}-0\right)=h_{n_{0}} .
$$

Если $x$ не совпадает ни с одной из точек $x_{n}$, то в этой точке функция $f(x)$ непрерывна. Функция $f(x)$ назьвается функцией скачков.

Зададим меру $\nu$ на $R$ в виде

$$
\nu\{(-\infty, x)\}=f(x)
$$

и будем считать, что

$$
\sum_{n} h_{n}=1, \quad h_{n}=h_{-n}, \quad n=0, \pm 1, \pm 2, \ldots .
$$

Если положить $x_{n}=n$, то

$$
\int_{R} \rho(u, s) \rho(u, t) d \nu(u)=\sum_{n=-\infty}^{\infty} h_{n} \rho(n, s) \rho(n, t) .
$$

Разложив корреляционную функцию в ряд по полной системе ортонормированных собственных функций

$$
B(t, s)=\sum_{n=-\infty}^{+\infty} \lambda_{n} \varphi_{n}(t) \varphi_{n}(s)
$$

видим, что все условия теоремы 1 будут выполнены, если положить

$$
\rho(u, t)= \begin{cases}0 & \text { для }-1<u<1, \\ \sqrt{\frac{\lambda_{n}}{2 h_{n}}} \varphi_{n}(t) & \text { для } u \in[n, n+1), \\ -\sqrt{\frac{\lambda_{n}}{2 h_{n}}} \varphi_{n}(t) & \text { для } u \in(-n-1,-n], \quad n=1,2, \ldots .\end{cases}
$$

В случае абсолютно непрерывной меры $\nu$ решение уравнения (7) было получено для винеровской меры, условной меры Винера и ряда других мер. Для меры Боголюбова можно построить следуюшее решение уравнения (7). Выберем в качестве $\nu$ нормированную меру Лебега на отрезке $[-\beta, \beta]$, т.е.

$$
d \nu(u)=\frac{1}{2 \beta} d u .
$$


Тогда

$$
\rho(u, t)=\sqrt{\frac{\beta}{m}} \frac{1}{e^{\beta \omega}-1} e^{\omega(t-|u|)}\left[\theta(t-|u|)+e^{\beta \omega} \theta(|u|-t)\right] \operatorname{sign} u .
$$

Можно проверить, что так выбранные мера $\nu$ и функция $\rho(u, t)$ удовлетворяют всем условиям теоремы 1.

Итак, в рассматриваемом случае боголюбовской меры имеем по теореме 1

$$
\int_{X} F(x) d \mu_{\mathrm{B}}(x) \approx \frac{1}{(2 \beta)^{n}} \int_{-\beta}^{\beta} \ldots \int_{-\beta}^{\beta} d u_{1} \ldots d u_{n} F\left(\sum_{j=1}^{n} c_{j} \rho\left(u_{j}, t\right)\right),
$$

где функция $\rho(u, t)$ задана формулой (10).

Рассмотрим приближенные формулы, точные для функциональных многочленов третьей степени и для функционалов специального вида.

Пусть по-прежнему $X$ - пространство $C[a, b]$ непрерывных на отрезке $[a, b]$ функций, $\nu$ - некоторая мера борелевских множеств действительной оси $R, A(u)$ - положительная на $R$ функция, $p(x)$ - весовой функционал. Предположим, что выполнены условия

$$
\begin{gathered}
\int_{R} A(u) d \nu(u) \equiv A<\infty \\
\int_{X} p(x) x(t) d \mu(x)=\int_{X} p(x) x(t) x(s) x(\tau) d \mu(x) \equiv 0, \\
r(t, s) \equiv \frac{1}{p_{0}} \int_{X} p(x) x(t) x(s) d \mu(x)<\infty
\end{gathered}
$$

где

$$
p_{0} \equiv \int_{X} p(x) d \mu(x)
$$

Tеорема 3 ([25]). Пусть симметричная функиия $r(t, s)$ представима в виде

$$
r(t, s)=\int_{R} x(u, t) x(u, s) d \nu(u)
$$

где функиия $x(u, t)$ принадлежит пространству $L_{2}[R, \nu]$ относительно арәумента и. Тогда формула

$$
\begin{aligned}
\int_{X} p(x) F(x) d \mu(x) \approx p_{0} & (1-A) F(0)+ \\
& +\frac{1}{2} p_{0} \int_{R} A(u)\left[F\left(\frac{x(u, \cdot)}{\sqrt{A(u)}}\right)+F\left(-\frac{x(u, \cdot)}{\sqrt{A(u)}}\right)\right] d \nu(u)
\end{aligned}
$$

точна, когда $F(x)$ - произвольный функииональный многочлен третьей степени.

Если мера $\nu$ дискретна, то

$$
A=\sum_{k} A_{k}, \quad r(t, s)=\sum_{k} x_{k}(t) x_{k}(s),
$$


и формула (11) принимает вид

$$
\int_{X} p(x) F(x) d \mu(x) \approx p_{0}(1-A) F(0)+\frac{p_{0}}{2} \sum_{k} A_{k}\left[F\left(\frac{x_{k}(\cdot)}{\sqrt{A_{k}}}\right)+F\left(-\frac{x_{k}(\cdot)}{\sqrt{A_{k}}}\right)\right] .
$$

Рассмотрим пример. Пусть вес

$$
p(x)=\int_{a}^{b} x^{2}(t) d t
$$

Тогда

$$
r(t, s)=B(t, s)+\frac{2}{\operatorname{tr} B} \int_{a}^{b} B(t, \tau) B(\tau, s) d \tau, \quad \operatorname{tr} B=\int_{a}^{b} B(t, t) d t .
$$

Если теперь воспользоваться разложением корреляционной функции в ряд по ее собственным функциям, то для функции $r(t, s)$ получим

$$
r(t, s)=\sum_{k}\left(\lambda_{k}+\frac{2}{\operatorname{tr} B} \lambda_{k}^{2}\right) \varphi_{k}(t) \varphi_{k}(s),
$$

где $\lambda_{k}$ - собственные числа ядра $B(t, s)$. Формула (12) примет вид

$$
\begin{aligned}
& \int_{X}\left(\int_{a}^{b} x^{2}(t) d t\right) F(x) d \mu(x) \approx \\
& \quad \approx \operatorname{tr} B\left\{(1-A) F(0)+\frac{1}{2} \sum_{k} A_{k}\left[F\left(b_{k} \varphi_{k}(\cdot)\right)+F\left(-b_{k} \varphi_{k}(\cdot)\right)\right]\right\},
\end{aligned}
$$

где

$$
b_{k}=\left[\frac{1}{A_{k}}\left(\lambda_{k}+\frac{2}{\operatorname{tr} B} \lambda_{k}^{2}\right)\right]^{\frac{1}{2}}, \quad \sum_{k} A_{k}=A<\infty .
$$

TeOPEмa 4 ([25]). Пусть функиии $r(t, s) u$

$$
\rho(t, s)=\frac{1}{p_{0}} \int_{X} p(x) V(x) x(t) x(s) d \mu(x),
$$

әде $V(x)$ - некоторый четный положительный на $C$ функционал, представимы в виде

$$
r(t, s)=\sum_{k} x_{k}(t) x_{k}(s), \quad \rho(t, s)=\sum_{k} B_{k} x_{k}(t) x_{k}(s),
$$

где $B_{k}$ - такие величины, что при каждом значении $k$ уравнение

$$
V\left(\frac{x_{k}(\cdot)}{\sqrt{A_{k}}}\right)=B_{k}
$$

имеет положительное решение $A_{k}$ такое, что

$$
A=\sum_{k} A_{k}<\infty
$$


Тогда формула (12) точна для всех функциональных многочленов третьей степени, а также для функционалов $F(x)$ вида

$$
F(x)=V(x) p_{2}(x)
$$

где $p_{2}(x)$ - произвольный однородный функиионал второй степени.

В качестве примера рассмотрим случай меры Боголюбова. Пусть

$$
p(x) \equiv 1, \quad V(x)=\|x\|^{2}=\int_{0}^{\beta} x^{2}(t) d t .
$$

Тогда

$$
\rho(t, s)=\sum_{k} B_{k} x_{k}(t) x_{k}(s)
$$

где $B_{k}=\operatorname{tr} B+2 \lambda_{k}, x_{k}(t)=\sqrt{\lambda_{k}} \varphi_{k}(t)$, а $\lambda_{k}$ и $\varphi_{k}(t)$ - собственные значения и собственные функции ядра $B(t, s)$. Другие величины, входящие в формулу (12), в рассматриваемом случае задаются соотношениями

$$
\begin{gathered}
\operatorname{tr} B=\frac{\beta}{2 m \omega} \operatorname{cth} \frac{\beta \omega}{2}, \quad A_{k}=\left(2+\frac{\beta}{2 \omega} \operatorname{cth} \frac{\beta \omega}{2}\left[\omega^{2}+\left(2 \pi k \beta^{-1}\right)^{2}\right]\right)^{-1}, \\
A=\frac{1}{\sqrt{1+\frac{4}{\beta \omega} \operatorname{th} \frac{\beta \omega}{2}}} \frac{\operatorname{cth}\left(\frac{\beta \omega}{2} \sqrt{1+\frac{4}{\beta \omega} \operatorname{th} \frac{\beta \omega}{2}}\right)}{\operatorname{cth} \frac{\beta \omega}{2}} .
\end{gathered}
$$

\section{5. НЕРАВЕНСТВА ДЛЯ РАВНОВЕСНЫХ СРЕДНИХ}

Рассмотрим систему с гамильтонианом $\widehat{H}=\widehat{\Gamma}+\widehat{V}$, где $\widehat{V}=V(\hat{q})$ - взаимодействие, а также однопараметрическое семейство гамильтонианов

$$
\widehat{H}(h)=\widehat{\Gamma}(h)+\widehat{V}, \quad \widehat{\Gamma}(h)=\frac{\hat{p}^{2}}{2 m}+\frac{m \omega^{2}}{2}(\hat{q}-h)^{2}, \quad h \in R .
$$

Статсумма рассматриваемой системы $Z(h)=\operatorname{tr} e^{-\beta \widehat{H}(h)}$ после канонического преобразования $\hat{q}-h \rightarrow \hat{q}$ принимает вид

$$
Z(h)=\operatorname{tr} \exp \{-\beta[\widehat{\Gamma}+V(\hat{q}+h)]\} .
$$

Будем предполагать, что потенциал взаимодействия неотрицателен и симметричен, т.е. $V(x) \geqslant 0, V(x)=V(-x)$. С помощью оператора хронологического упорядочения можно записать [15], что

$$
e^{-\beta(\widehat{\Gamma}+\widehat{V})}=e^{-\beta \widehat{\Gamma}} T \exp \left(-\int_{0}^{\beta} d s e^{s \widehat{\Gamma}} \widehat{V} e^{-s \widehat{\Gamma}}\right)
$$

Тогда

$$
R(h) \equiv \frac{\operatorname{tr} e^{-\beta \widehat{H}(h)}}{\operatorname{tr} e^{-\beta \widehat{\Gamma}}}=\left\langle T \exp \left[-\int_{0}^{\beta} d s V(\widehat{Q}(s)+h)\right]\right\rangle_{\widehat{\Gamma}} .
$$


Записывая соотношение (13) через боголюбовский функциональный интеграл, имеем

$$
R(h)=\int_{X} \exp \left[-\int_{0}^{\beta} d s V(x(s)+h)\right] d \mu_{\mathrm{Б}}(x) .
$$

Воспользуемся теперь теоремой о линейной замене переменной в интеграле по гауссовой мере [20], которая для интегрируемого функционала $F(x)$ и функции $a \in H$ дает

$$
\int_{X} F(x) d \mu(x)=e^{-\frac{1}{2}\|a\|_{H}^{2}} \int_{X} F(x+a) e^{-(a, x)} d \mu(x) .
$$

Применим формулу (14) для случая меры Боголюбова и для постоянных функций $a$, которые в этом случае принадлежат $H$. Тогда имеем

$$
\int_{X} F(x+a) d \mu_{\text {Б }}(x)=\exp \left\{-\frac{\beta m \omega^{2} a^{2}}{2}\right\} \int_{X} F(x) \exp \left\{a m \omega^{2} \int_{0}^{\beta} x(t) d t\right\} d \mu_{\text {Б }}(x) .
$$

$\mathrm{C}$ помощью последнего соотношения функция $R(h)$ может быть записана в виде

$$
R(h)=\exp \left\{-\frac{\beta m \omega^{2} h^{2}}{2}\right\} \int_{X} \exp \left\{-\int_{0}^{\beta} V(x(t)) d t\right\} \exp \left\{m h \omega^{2} \int_{0}^{\beta} x(t) d t\right\} d \mu_{\text {Б }}(x) .
$$

Рассмотрим теперь преобразование Фурье-Гаусса функционала $f(x)$,

$$
\tilde{f}(y) \equiv F(f ; y)=\int_{X} f(x+i y) d \mu_{\mathrm{Б}}(x),
$$

и равенство Парсеваля

$$
\int_{X} f\left(\frac{x}{\sqrt{2}}\right) g^{*}\left(\frac{x}{\sqrt{2}}\right) d \mu_{\mathrm{Б}}(x)=\int_{X} F\left(f ; \frac{y}{\sqrt{2}}\right) F^{*}\left(g ; \frac{y}{\sqrt{2}}\right) d \mu_{\mathrm{Б}}(y)
$$

для случая функционалов

$$
f(x)=F(x) \equiv \exp \left\{-\int_{0}^{\beta} d t V(x(t))\right\}, \quad g(x)=\exp \left\{m h \omega^{2} \int_{0}^{\beta} x(t) d t\right\} .
$$

Равенство (15) принимает вид

$$
\begin{gathered}
\exp \left\{-\frac{\beta m h^{2} \omega^{2}}{2}\right\} \int_{X} F\left(\frac{x}{\sqrt{2}}\right) \exp \left\{\frac{1}{\sqrt{2}} h m \omega^{2} \int_{0}^{\beta} x(t) d t\right\} d \mu_{\mathrm{Б}}(x)= \\
=\int_{X} \widetilde{F}\left(\frac{y}{\sqrt{2}}\right) \exp \left\{\frac{i}{\sqrt{2}} h m \omega^{2} \int_{0}^{\beta} y(t) d t\right\} d \mu_{\mathrm{Б}}(y),
\end{gathered}
$$

откуда видно, что если при любом $y$ выполняется неравенство

$$
\widetilde{F}(y) \geqslant 0,
$$

TO

$$
R(h)=\widetilde{F}(-i h) \leqslant R(0)=\widetilde{F}(0)
$$


Условие (16) доказывается следующим образом. Для симметричных потенциалов имеeм

$$
\begin{aligned}
\widetilde{F}(y) & =e^{\frac{1}{2}(y, y)} \int_{X} \exp \left\{-\int_{0}^{\beta} V(x) d t+i(x, y)\right\} d \mu_{\mathrm{Б}}(x)= \\
& =e^{\frac{1}{2}(y, y)} \int_{X} \exp \left\{-\int_{0}^{\beta} V(x) d t-i(x, y)\right\} d \mu_{\mathrm{Б}}(x)=\widetilde{F}^{*}(y),
\end{aligned}
$$

т.е. в этом случае преобразование Фурье-Гаусса действительно. Докажем, что оно неотрицательно. Учтем, что

$$
e^{\frac{1}{2}\|y\|^{2}} \int_{X} e^{i(x, y)} d \mu_{5}(x)=1
$$

и применим неравенство Иенсена. Тогда имеем

$$
\widetilde{F}(y) \geqslant \exp \left\{-e^{\frac{1}{2}\|y\|^{2}} \int_{X} d \mu_{\mathrm{Б}}(x) e^{i(x, y)} \int_{0}^{\beta} V(x(t)) d t\right\} \geqslant 0,
$$

что и завершает доказательство неравенства (16).

Из условия (17) следует, в частности, что

$$
(\hat{q}, \hat{q})_{\hat{H}} \leqslant \frac{1}{\beta m \omega^{2}},
$$

где внутреннее произведение Боголюбова произвольных операторов $\hat{A}$ и $\widehat{B}$ определяется как

$$
(\hat{A}, \widehat{B})_{\widehat{H}}=\frac{1}{\beta \operatorname{tr} e^{-\beta \hat{H}}} \int_{0}^{\beta} d s \operatorname{tr}\left[e^{-s \hat{H}} \hat{A} e^{-(\beta-s) \hat{H}} \widehat{B}\right]=(\widehat{B}, \hat{A})_{\hat{H}}
$$

Если перейти от операторов $\hat{q}, \hat{p}$ к операторам $\hat{b}, \hat{b}^{\dagger}$ с помошью соотношений

$$
\hat{q}=\frac{1}{\sqrt{2 m \omega}}\left(\hat{b}+\hat{b}^{\dagger}\right), \quad \hat{p}=i \sqrt{\frac{m \omega}{2}}\left(\hat{b}^{\dagger}-\hat{b}\right)
$$

и учесть правила отбора для равновесных средних по квадратичному гамильтониану, то неравенство (18) примет вид

$$
\left(\hat{b}^{\dagger}, \hat{b}\right)_{\widehat{H}} \leqslant(\beta \omega)^{-1} \text {. }
$$

Из соотношения (18) можно получить неравенство для гиббсовского равновесного среднего $\left\langle\hat{q}^{2}\right\rangle_{\hat{H}}$. Для этого необходимо воспользоваться неравенством ФолькаБрука [26]. Пусть

$$
g=\left\langle\hat{q}^{2}\right\rangle_{\hat{H}}, \quad b=(\hat{q}, \hat{q})_{\hat{H}}, \quad c=\langle[\hat{q},[\beta \widehat{H}, \hat{q}]]\rangle_{\widehat{H}} .
$$

Пусть также имеют место оценки сверху $b \leqslant b_{0}, c \leqslant c_{0}$. Тогда

$$
g \leqslant g_{0} \equiv \frac{1}{2} \sqrt{c_{0} b_{0}} \text { cth } \sqrt{\frac{c_{0}}{4 b_{0}}} .
$$

В рассматриваемом случае $b_{0}=\left(\beta m \omega^{2}\right)^{-1}, c_{0}=\beta / m$, и последнее неравенство дает

$$
\left\langle\hat{q}^{2}\right\rangle_{\hat{H}} \leqslant \frac{1}{2 m \omega} \operatorname{cth} \frac{\beta \omega}{2}=\left\langle\hat{q}^{2}\right\rangle_{\widehat{\Gamma}} .
$$

Условие (17) является примером так называемого условия гауссовой доминантности [27], а вытекаюшее из (17)условие (18) - примером так называемого условия локаль- 
ной гауссовой доминантности [28], играющего важную роль в теории фазовых переходов. Оценка типа оценки (19) ранее была получена в менее общем случае одномерного нелинейного осциллятора в работе [29].

Благодарности. Работа поддержана Р $\Phi И$, проект № 99-01-00887.

\section{Список литературы}

[1] M. Fréchet. Bull. Soc. Math. France. 1915. V. 43. P. 249.

[2] P. J. Daniell. Ann. Math. 1917-1918. V. 19. P. 279.

[3] P. J. Daniell. Ann. Math. 1918-1919. V. 20. P. 281.

[4] Л. Люмис. Введение в абстрактный гармонический анализ. М.: ИЛ, 1956.

[5] Ф. Рисс, Б. Секефальви-Надь. Лекции по функциональному анализу. М.: Мир, 1979.

[6] N. Wiener. Ann. Math. 1920. V. 22. P. 66.

[7] R. Feynman. Rev. Mod. Phys. 1948. V. 20. P. 367.

[8] R. Cameron. J. Anal. Math. 1962/63. V. 10. P. 287.

[9] S. F. Edwards, R. E. Pierls. Proc. Roy. Soc. 1954. V. A224. P. 24.

[10] K. Symanzik. Zs. f. Naturforsch. 1954. V. 9a. P. 809.

[11] И. М. Гельфанд, Р. А. Минлос. ДАН СССР. 1954. Т. 97. С. 209.

[12] Е. С. Фрадкин. ДАН СССР. 1954. Т. 98. С. 47.

[13] Н. Н. Боголюбов. ДАН СССР. 1954. Т. 99. С. 225.

[14] А. В. Свидзинский. ЖЭТФ. 1956. Т. 31. С. 324.

[15] Н. Н. Боголюбов, Н. Н. Боголюбов (мл.). Аспекты теории полярона. Сообщения ОИЯИ, P17-81-65. Дубна: ОИЯИ, 1981.

[16] Д. П. Санкович. ТМФ. 1999. Т. 119. С. 345.

[17] Х.-С. Го. Гауссовские меры в банаховых пространствах. М.: Мир, 1979.

[18] Г. Е. Шилов, Фан Дык Тинь. Интеграл, мера и производная на линейных пространствах. М.: Наука, 1967.

[19] А. Д. Егоров, Л. А. Янович. ДАН БССР. 1970. Т. 14. С. 873.

[20] Л. А. Анович. Приближенное вычисление континуальных интегралов по гауссовым мерам. Минск: Наука и техника, 1976.

[21] J. Kuelbs. J. Funct. Anal. 1970. V. 5. P. 354.

[22] B. S. Rajput. J. Multivar. Anal. 1972. V. 2. P. 282.

[23] L. Gross. Abstract Wiener spaces. In: Proc. 5th Berkeley Symp. Math. Stat. and Prob. 1965. V.2. Eds. L. M. LeCam, J. Neyman. Berkeley: Univ. of California Press, 1967. P. 31.

[24] M. Kac. On some connections between probability theory and differential and integral equations. In: Proc. 2nd Berkeley Symp. Math. Stat. and Prob. Ed. J. Neyman. Berkeley: Univ. of California Press, 1951. P. 189

[25] П. И. Соболевский, Л. А. Янович. ДАН БССР. 1974. Т. 18. С. 965.

[26] H. Falk, L. W. Bruch. Phys. Rev. 1969. V. 180. P. 442.

[27] J. Fröhlich. Bull. Amer. Math. Soc. 1978. V. 84. P. 165.

[28] Д. П. Санкович. ТМФ. 1989. Т. 79. С. 460.

[29] N. N. Bogolubov, Jr., D. P. Sankovich. Phys. Lett. A. 1989. V. 137. P. 179.

Поступила в редакцию 25.V.2000 г. 